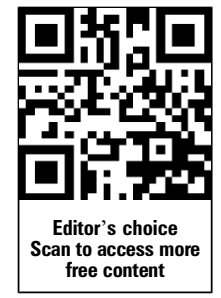

- Additional material is published online only. To view please visit the journal online (http://dx.doi.org/10.1136/ heartjnl-2012-303329).

${ }^{1}$ Department of Cardiology, Wroclaw Medical University, Wroclaw, Poland

${ }^{2}$ School of Medicine, University of Queensland, Brisbane, Australia

${ }^{3}$ Menzies Research Institute of Tasmania, Hobart, Australia

\section{Correspondence to} Dr Thomas H Marwick, Menzies Research Institute of Tasmania, 17 Liverpool St, Hobart, Tas 7000, Australia; tom.marwick@utas.edu.au

Received 12 November 2012 Revised 17 December 2012 Accepted 20 December 2012 Published Online First 23 January 2013
To cite: Kosmala W Przewlocka-Kosmala M, Szczepanik-Osadnik $\mathrm{H}$, et al. Heart 2013;99:320-326.

\title{
Fibrosis and cardiac function in obesity: a randomised controlled trial of aldosterone blockade
}

Wojciech Kosmala, ${ }^{1}$ Monika Przewlocka-Kosmala, ${ }^{1}$ Hanna Szczepanik-Osadnik, ${ }^{1}$ Andrzej Mysiak, ${ }^{1}$ Thomas H Marwick ${ }^{2,3}$

\section{ABSTRACT \\ Objectives As myocardial fibrosis might be an} important contributor to the association of obesity with left ventricular (LV) dysfunction and heart failure, we investigated the effects of spironolactone on LV function and serological fibrosis markers (procollagen type III Nterminal propeptide (PIIINP) and procollagen type I Cterminal propeptide (PICP)) in patients with obesity and abnormal LV performance.

Design A prospective, randomised, double-blind, placebo-controlled study.

Setting A university hospital.

Patients and intervention 113 patients (mean \pm SD age $58 \pm 8$ years) with body mass index $\geq 30$, without any comorbidities, with impaired early diastolic mitral annular velocity, randomised to spironolactone $25 \mathrm{mg} /$ day or placebo for 6 months.

Main outcome measures Echocardiographically derived indices of LV systolic (strain and strain rate) and diastolic (E velocity, tissue $\mathrm{e}^{\prime}$ and $E / \mathrm{e}^{\prime}$ ratio) function, myocardial reflectivity (calibrated integrated backscatter (IB)), and serum PICP and PIIINP.

Results In the spironolactone group, significant improvements in myocardial deformation, peak early diastolic velocity (Em), E/e' and IB were noted with a simultaneous decrease in PICP and PIIINP. No corresponding alterations were found with placebo. Improvement in LV systolic function (increase in strain) was independently associated with baseline strain $(\beta=-0.43, p<0.001)$, change in $\mathrm{IB}(\beta=0.26, p<0.02)$ and baseline PICP $(\beta=0.24, p<0.04)$. Among the independent determinants of LV diastolic improvement were for increase in Em - baseline $\mathrm{Em}(\beta=-0.44$, $p<0.001)$ and baseline PICP $(\beta=0.35, p<0.002)$, and for decrease in $E / e^{\prime}-$ baseline $E / e^{\prime}(\beta=-0.35, p<0.005)$ and change in PICP $(\beta=0.25, p<0.04)$.

Conclusions In patients with obesity without other comorbidities, aldosterone antagonism improves LV function and myocardial acoustic properties, and reduces circulating procollagen levels. Beneficial changes in cardiac performance are independently predicted by baseline LV dysfunction and baseline disturbances, as well as treatment-induced improvements in fibrosis markers.

Clinical Trial Registration http://www.anzctr.org.au ACTRN12609000655246.

\section{INTRODUCTION}

Left ventricular (LV) dysfunction and heart failure in obese subjects, developing independently of commonly coexisting coronary artery disease, hypertension and diabetes mellitus, are serious complications that contribute to increased morbidity and mortality. ${ }^{1-7}$ Given the difficulties associated with the achievement of sustained reduction of body mass, other preventive and therapeutic measures to decrease the risk of heart failure development in this population are becoming particularly relevant.

Growing evidence indicates that myocardial fibrosis is one of the pivotal contributors to heart muscle dysfunction in obesity. ${ }^{8-11}$ Previous studies have shown a benefit from aldosterone antagonism (AA) in patients with heart failure, associated with the limitation of collagen turnover in different stages of cardiac decompensation, also including mildly symptomatic subjects. ${ }^{12-15}$ Our previous work in patients with the metabolic syndrome showed a favourable effect of AA on cardiac function and morphology, when used in addition to background therapy with ACE inhibitors or angiotensin receptor blockers. ${ }^{16}$ However, it has not been established whether this benefit is merely linked to the coexistence of hypertension and diabetes or could represent an effect on the profibrotic properties of adiposity, independent of these comorbidities.

In view of this, we postulated that the addition of AA to the therapy of patients with excess weight and LV dysfunction might improve myocardial performance due to a reduction in fibrosis intensity. To test this hypothesis, we investigated the effect of 6 months of treatment with spironolactone on LV function parameters and serological markers of fibrosis (procollagen type I C-terminal propeptide (PICP) and procollagen type III N-terminal propeptide (PIIINP)) in obese patients without other comorbidities, in whom cardiac functional abnormalities were found.

\section{METHODS}

\section{Study design}

This report follows the recommendations of the 2010 Consolidated Standards of Reporting Trials Statement. ${ }^{17}$ The present study was designed as a prospective, blinded, parallel-group, placebocontrolled trial assessing the ability of 6 months of therapy with spironolactone $25 \mathrm{mg} /$ day to improve myocardial performance and morphology and reduce fibrosis intensity in patients with obesity. The primary end point was LV diastolic function (myocardial peak early diastolic velocity (Em)), with secondary imaging end points being LV systolic function (strain and peak systolic strain rate) and filling pressure (the ratio of mitral inflow early 
diastolic velocity to peak early diastolic mitral annular velocity $\left(\mathrm{E} / \mathrm{e}^{\prime}\right)$ ), myocardial reflectivity (evaluated by integrated backscatter (IB)), LV wall thicknesses and LV mass. Non-imaging secondary end points comprised alterations in collagen turnover as expressed by serum levels of PICP and PIIINP.

The sample size for addressing the primary end point was calculated on the basis of changes in diastolic variables in previous studies involving patients treated with $\mathrm{AA}$, as well as our pilot data. ${ }^{12} 16$ Assuming a $15 \%$ improvement with treatment and applying the variance seen in our patients, we anticipated a sample size of 60 per group to show a significant difference $(p<0.05)$ at a power of $90 \%$. To allow for drop-outs, the number of patients in each study arm was increased to 63 .

The procedure of randomisation to spironolactone or matching placebo was performed in blocks of 10 with an allocation ratio 1:1 using sequentially numbered, opaque, sealed envelopes. The designated person coordinating the study, who was not involved in field procedures, was responsible for the preparation of the randomisation list and the study drugs. Patients participating in the study and the investigators evaluating the outcomes were blinded to group assignment. Study medication was to be withdrawn on the occurrence of significant hyperkalaemia $(>5.5 \mathrm{mmol} / \mathrm{l})$ or serious side effects.

Ethics approval was obtained in Brisbane and Wroclaw, and informed consent was obtained from all patients. The study was registered with the Australian and New Zealand Clinical trial registry. Recruitment was only conducted at Wroclaw because the senior investigator (TM) moved from his original position.

\section{Patient selection}

Of a total of 356 obese men and women (body mass index (BMI) $>30 \mathrm{~kg} / \mathrm{m}^{2}$ ) initially analysed, who were recruited from the community as part of the health screening programme, we included 126 consecutive subjects satisfying the selection criteria over the period from August 2009 to November 2010 (see online supplementary figure), and followed them for 6 months to examine the response to therapy. Patients with previously diagnosed cardiovascular disease were not considered as candidates for the study.

All participants were subjected to a medical evaluation, comprising clinical history, routine blood analysis, lipid profile, a $75 \mathrm{~g}$ oral glucose tolerance test, ECG and echocardiography. The inclusion criterion was LV function abnormality defined as peak early diastolic velocity obtained from the septal part of the mitral annulus $<7 \mathrm{~cm} / \mathrm{s}$.

Ischaemic heart disease was excluded on the basis of negative history and normal stress echocardiography. Other exclusion criteria were: hypertension, diabetes mellitus, moderate and severe valvular heart diseases, absence of stable sinus rhythm, adrenocortical disorders, pulmonary, hepatic, rheumatic, neoplastic, skeletal, thyroid and renal diseases (including renal insufficiency with serum creatinine $>132 \mu \mathrm{mol} / \mathrm{l}(1.5 \mathrm{mg} / \mathrm{dl}))$, hyperkalaemia $>5.0 \mathrm{mmol} / \mathrm{l}$ and chronic use of any drugs.

\section{Study protocol}

Baseline evaluation included physical examination (with blood pressure measurement and anthropometric assessment including height, body weight, and both hip and waist circumference), blood sampling for laboratory measurements, dual energy x-ray absorptiometry for body fat estimation, ECG and echocardiography. Subsequently, patients were randomised to be treated with either $25 \mathrm{mg}$ spironolactone or placebo once daily. Control visits for the evaluation of patients' status, compliance with therapy and serum electrolytes were scheduled every 2 weeks during the first 2 months and then monthly. After 6 months, the baseline investigations were repeated.

\section{Blood assays}

Peripheral venous blood samples were obtained between 08:00 and 09:00 $\mathrm{h}$ in the morning after a $30 \mathrm{~min}$ rest in the supine position. Serum PICP concentrations were estimated with ELISA kits manufactured by Takara Biochemicals (Osaka, Japan); intra- and inter-assay coefficients of variation were $<3 \%$ and $<7 \%$. Serum PIIINP levels were measured by a radioimmunoassay technique using commercially available kits from Orion Diagnostica (Espoo, Finland); intra- and inter-assay coefficients of variation were $3.9 \%$ and $5.3 \%$. Serum transforming growth factor $\beta_{1}$ (TGF- $\left.\beta_{1}\right)$ levels were quantified using ELISA kits produced by R\&D Systems, (Minneapolis, Minnesota, USA); intra- and inter-assay coefficients of variation were $2.7 \%$ and $7.0 \%$. Serum aldosterone was assessed by a radioimmunoassay from Immunotech SAS (Marseille, France); intra- and inter-assay coefficients of variation were $7.6 \%$ and $8.8 \%$. Plasma angiotensin II levels were measured by radioimmunoassay using kits produced by DIAsource ImmunoAssays (Nivelles, Belgium); intra- and inter-assay coefficients of variation were $3.1 \%$ and $4.3 \%$.

\section{Evaluation of body composition}

Body composition was assessed by dual energy x-ray absorptiometry (Lunar DPX-plus; Lunar Corporation, Madison, Wisconsin, USA). Scans were carried out according to the manufacturer's instructions with subjects in the supine position. Abdominal fat content (android fat deposit) was estimated in the region of interest extending from the upper edge of the second lumbar vertebra to the lower edge of the fourth lumbar vertebra. Gynoid fat deposit was determined in the region of interest bounded superiorly by the upper part of the trochanter major of the femur and inferiorly by the bottom of the knees.

\section{Echocardiography}

Echocardiographic imaging was performed using Vivid 7 and System 5 equipment (GE; Vingmed Ultrasound, Horten, Norway) with phased array $2.5 \mathrm{MHz}$ multifrequency transducers.

\section{Conventional echocardiography}

The measurements of cardiac dimensions and wall thicknesses were carried out according to standard recommendations. ${ }^{18} \mathrm{LV}$ ejection fraction was computed using a modified Simpson's biplane method. Peak early (E) and late diastolic flow velocity (A), and deceleration time of early diastolic flow wave (DT) were measured from pulsed wave Doppler recordings of the LV inflow.

\section{Tissue Doppler imaging}

To evaluate LV longitudinal function, real time colour Doppler myocardial imaging data were acquired in the three apical views. The narrowest possible image sector angle and the optimal depth of imaging were used to maximise the frame rate. The sampling window was placed as parallel as possible to the myocardial segment of interest to ensure that the insonation angle did not exceed $20^{\circ}$. The region of interest was located in the central part of each segment interrogated and upheld at the same position during the heart cycle by manual tracking. Digital data were saved in a cine-loop format and subsequently analysed using an off-line workstation (GE; Echopac, Horten, Norway). 


\section{Myocardial velocities}

To evaluate peak systolic velocity $(\mathrm{Sm})$ and Em, regional myocardial velocity curves were derived from colour Doppler data acquired in the basal segment of the interventricular septum.

The ratio of mitral inflow early diastolic velocity to the average peak early diastolic mitral annular velocity achieved by pulsed-wave tissue Doppler from the septal and lateral portions of the mitral annulus $\left(\mathrm{E} / \mathrm{e}^{\prime}\right)$ was calculated for LV filling pressure approximation.

\section{Myocardial deformation}

LV myocardial deformation curves were obtained from the apical, mid and basal segments of each LV wall. Strain rate was assessed from the spatial velocity gradient over a computation distance of $12 \mathrm{~mm}$. The analysis of LV deformation curves included peak strain, defined as the greatest negative value on the strain curve, and peak systolic strain rate.

\section{Calibrated IB}

IB curves were obtained in the parasternal long-axis view. Measurements were performed by positioning a $9 \times 9$ pixel sample volume in the basal septum, posterior wall, and pericardium in end diastole. Calibrated IB was calculated by subtracting average pericardial IB intensity from average myocardial IB intensity of the septum and posterior wall.

All strain, strain rate, myocardial velocity and IB profiles were averaged from three consecutive cardiac cycles. The results for strain and strain rate are shown as the average values from all segments undergoing evaluation. All off-line analyses were performed by an observer blinded to the patients' clinical data.

\section{Feasibility}

The reproducibility of myocardial measurements was assessed using values averaged from all the segments analysed in 15 randomly selected examinations. The intra- and inter-observer variability was $0.7 \pm 0.4 \%$ and $0.8 \pm 0.5 \%$ for strain, $0.05 \pm 0.02$ and $0.07 \pm 0.04 \mathrm{~s}^{-1}$ for strain rate, $0.2 \pm 0.3$ and $0.3 \pm 0.3 \mathrm{~cm} / \mathrm{s}$ for myocardial velocity, and $1.2 \pm 1.3$ and $1.4 \pm 1.4 \mathrm{~dB}$ for calibrated $\mathrm{IB}$, respectively.

Baseline fibrosis intensity was estimated in obese patients on the basis of comparison with serum levels of PICP and PIIINP assessed in a group of 34 age-matched healthy volunteers (57.1 \pm 8.5 years) with normal BMI and cardiac function and morphology.

\section{Statistical analysis}

Data are presented as mean \pm SD for normal and median (IQR) for skewed distribution. Between-group comparisons were performed by an unpaired two-sided Student $t$ test or, when more than two groups were included, by one-way analysis of variance with Scheffe's post hoc test for continuous variables and by $\chi^{2}$ for categorical variables. Homogeneity of variances was evaluated by the Levene test. Longitudinal analyses were carried out using a repeated measures two-way analysis of variance with Tukey's HSD post hoc test. Associations between variables were expressed with Pearson or Spearman correlation coefficients and stepwise multiple regression analysis. Skewed variables were analysed after the log-transformation. Effect size was evaluated using the Cohen's d method. Changes in particular variables with intervention were calculated by subtracting the baseline value from the follow-up value and were expressed in the units of their measurements. All analyses were performed on a per protocol basis, with standard statistical software (Statistica for
Windows 10, StatSoft, Tulsa, Oklahoma, USA). The level of statistical significance was set at a two-sided $\mathrm{p}$ value $<0.05$.

\section{RESULTS}

\section{Patient characteristics}

The baseline demographic, clinical and laboratory characteristics of both study arms are presented in table 1. Echocardiographic characteristics are summarised in table 2 . There were no significant differences between the treatment and placebo groups with respect to any variable. All the enrolees were asymptomatic or presented slight limitation of exercise tolerance, categorising them as New York Heart Association class I or II. The serological fibrosis markers were significantly higher in obese patients than healthy slim referents (PICP level in the controls was $118 \pm 41 \mu \mathrm{g} / \mathrm{l} ; \mathrm{p}<0.002$ vs the spironolactone and $\mathrm{p}<0.001$ vs the placebo group. PIIINP level in the controls was $4.4 \pm 1.0 \mu \mathrm{g} / 1 ; \mathrm{p}<0.03$ vs the spironolactone and $\mathrm{p}<0.04$ vs the placebo group).

\section{Patient compliance and drug side effects}

All planned patients were recruited (63 per group). Four patients, two allocated to the treatment group and two to the placebo group, did not complete the study protocol because of changing residence. Three patients from the spironolactone group and six from the placebo group refused to continue their participation in the study. Apart from mild gynaecomastia in one male patient in the spironolactone group, which did not require withdrawal of the drug, no adverse effects or complications were noted.

\section{Changes in clinical and laboratory variables with intervention}

There were no significant changes at follow-up in either group in all body-fat-related variables, plasma lipids, indices of renal function and metabolic control, C-reactive protein, circulating aldosterone and angiotensin II. The spironolactone group exhibited a significant decrease in blood pressure and a slight increase in serum potassium level (table 1).

A significant decrease in blood levels of the analytes associated with fibrosis (PICP, PIIINP and TGF- $\beta_{1}$ ) was shown in the intervention group, and no changes were noted with placebo (table 1). The effect size was found to be of intermediate magnitude ( $\mathrm{d}=0.70$ for PICP, 0.41 for PIIINP and 0.49 for TGF- $\beta_{1}$ ).

\section{Changes in cardiac function and morphology with intervention \\ LV function}

The treatment group showed improvements in LV systolic function (evidenced by an increase in strain, peak systolic strain rate and $\mathrm{Sm}$ ), as well as in LV diastolic function (reflected by an increase in Em and a decrease in $\mathrm{E} / \mathrm{e}^{\prime}$, table 2). The effect size was large for strain $(\mathrm{d}=1.80)$, peak systolic strain rate $(\mathrm{d}=1.44)$ and $\operatorname{Em}(\mathrm{d}=1.12)$, and medium for $\operatorname{Sm}(\mathrm{d}=0.59)$ and $\mathrm{E} / \mathrm{e}^{\prime}$ $(\mathrm{d}=0.45)$.

\section{Tissue characterisation}

Significant decrease in calibrated IB in the basal septum and posterior wall, indicative of the improvement in myocardial tissue echodensity, was noted with spironolactone therapy (table 2), and the effect was of intermediate size $(\mathrm{d}=0.44$ for the basal septum and 0.43 for the posterior wall). 
Table 1 Demographic data and baseline and follow-up values of clinical and laboratory characteristics of the study group

\begin{tabular}{|c|c|c|c|c|c|c|c|}
\hline \multirow[b]{2}{*}{ Characteristic } & \multicolumn{3}{|c|}{ Spironolactone $(n=58)$} & \multicolumn{3}{|c|}{ Placebo $(n=55)$} & \multirow{2}{*}{$\begin{array}{l}\text { p Value } \\
\Delta \text { Spironolactone } \\
\text { vs } \Delta \text { Placebo }\end{array}$} \\
\hline & Baseline & Follow-up & p Value & Baseline & Follow-up & p Value & \\
\hline Age, years & $58.8 \pm 8.9$ & & & $58.8 \pm 7.9$ & & & \\
\hline Male & $24(41)$ & & & $19(35)$ & & & \\
\hline BMI, $\mathrm{kg} / \mathrm{m}^{2}$ & $35.7 \pm 5.2$ & $35.6 \pm 5.2$ & 0.41 & $35.5 \pm 5.0$ & $35.2 \pm 4.9$ & 0.23 & 0.58 \\
\hline Waist circumference, $\mathrm{cm}$ & $106 \pm 15$ & $106 \pm 16$ & 0.67 & $105 \pm 13$ & $105 \pm 13$ & 0.36 & 0.35 \\
\hline Waist-to-hip ratio & $0.95 \pm 0.12$ & $0.95 \pm 0.12$ & 0.49 & $0.95 \pm 0.10$ & $0.95 \pm 0.10$ & 0.71 & 0.48 \\
\hline Systolic blood pressure, $\mathrm{mm} \mathrm{Hg}$ & $132 \pm 5$ & $128 \pm 6$ & 0.001 & $131 \pm 6$ & $130 \pm 6$ & 0.38 & 0.001 \\
\hline Diastolic blood pressure, $\mathrm{mm} \mathrm{Hg}$ & $80 \pm 4$ & $77 \pm 5$ & 0.001 & $79 \pm 5$ & $79 \pm 4$ & 0.54 & 0.004 \\
\hline Body fat content, $\mathrm{kg}$ & $33.3 \pm 7.7$ & $33.0 \pm 7.7$ & 0.58 & $32.8 \pm 6.8$ & $32.5 \pm 6.7$ & 0.22 & 0.84 \\
\hline Android fat deposit, $\mathrm{kg}$ & $3.6 \pm 1.0$ & $3.6 \pm 1.1$ & 0.94 & $3.8 \pm 0.8$ & $3.7 \pm 0.9$ & 0.44 & 0.28 \\
\hline Gynoid fat deposit, kg & $8.4 \pm 2.3$ & $8.3 \pm 2.4$ & 0.36 & $8.2 \pm 2.2$ & $8.1 \pm 2.2$ & 0.20 & 0.81 \\
\hline Android/gynoid fat & $0.45 \pm 0.13$ & $0.45 \pm 0.14$ & 0.88 & $0.49 \pm 0.18$ & $0.48 \pm 0.17$ & 0.66 & 0.43 \\
\hline Fasting insulin, mIU/l & $9.3(6.4-14.0)$ & $11.4(5.6-17.1)$ & 0.39 & $6.4(3.0-8.1)$ & $7.3(4.3-13.7)$ & 0.34 & 0.86 \\
\hline Fasting glucose, mg/dl & $97 \pm 10$ & $97 \pm 10$ & 0.76 & $96 \pm 11$ & $95 \pm 10$ & 0.68 & 0.61 \\
\hline HOMA-IR & $2.5(1.6-3.7)$ & $2.9(1.4-4.7)$ & 0.36 & $1.7(1.0-2.3)$ & $2.1(1.1-3.6)$ & 0.43 & 0.83 \\
\hline Creatinine, mg/dl & $0.87 \pm 0.20$ & $0.85 \pm 0.21$ & 0.25 & $0.85 \pm 0.17$ & $0.84 \pm 0.14$ & 0.48 & 0.74 \\
\hline Potassium, $\mathrm{mmol} / \mathrm{l}$ & $4.2 \pm 0.3$ & $4.5 \pm 0.3$ & 0.0001 & $4.3 \pm 0.4$ & $4.3 \pm 0.3$ & 0.45 & 0.001 \\
\hline Total cholesterol, mg/dl & $208 \pm 44$ & $209 \pm 42$ & 0.85 & $216 \pm 44$ & $210 \pm 40$ & 0.38 & 0.42 \\
\hline Low-density lipoprotein, mg/dl & $127 \pm 38$ & $128 \pm 36$ & 0.86 & $134 \pm 39$ & $131 \pm 38$ & 0.61 & 0.61 \\
\hline High-density lipoprotein, mg/dl & $48 \pm 14$ & $48 \pm 13$ & 0.78 & $51 \pm 15$ & $49 \pm 15$ & 0.22 & 0.48 \\
\hline Triglycerides, mg/dl & $161 \pm 76$ & $166 \pm 80$ & 0.71 & $160 \pm 79$ & $152 \pm 88$ & 0.56 & 0.78 \\
\hline CRP, mg/l & $3.1(2.2-4.8)$ & $3.4(2.1-5.1)$ & 0.29 & $2.8(2.2-4.1)$ & $3.6(2.3-4.2)$ & 0.34 & 0.69 \\
\hline PIIINP, $\mu g / l$ & $5.2 \pm 2.0$ & $4.7 \pm 1.9$ & 0.04 & $5.2 \pm 2.0$ & $5.4 \pm 2.9$ & 0.62 & 0.03 \\
\hline PICP, $\mu \mathrm{g} / \mathrm{l}$ & $175 \pm 76$ & $146 \pm 71$ & 0.001 & $184 \pm 75$ & $190 \pm 79$ & 0.65 & 0.001 \\
\hline TGF- $\beta_{1}, \mu \mathrm{g} / \mathrm{ml}$ & $29.2 \pm 11.3$ & $26.2 \pm 9.2$ & 0.04 & $25.9 \pm 10.1$ & $26.8 \pm 10.2$ & 0.36 & 0.02 \\
\hline Aldosterone, pg/ml & $52(27-112)$ & $81(28-160)$ & 0.07 & $55(47-76)$ & $55(41-83)$ & 0.68 & 0.06 \\
\hline Angiotensin II, pmol/l & $10.7 \pm 4.7$ & $11.9 \pm 7.9$ & 0.54 & $14.0 \pm 5.2$ & $13.6 \pm 6.8$ & 0.74 & 0.25 \\
\hline
\end{tabular}

Values are mean \pm SD, number (\%) or median (IQR). $\Delta$ Placebo indicates change in the placebo group during follow-up, and $\Delta$ Spironolactone the change in the spironolactone group during follow-up.

BMI, body mass index; CRP, C-reactive protein; HOMA-IR, homoeostasis model of assessment-insulin resistance; PICP, procollagen type I C-terminal propeptide; PIIINP, procollagen type III N-terminal propeptide; TGF, transforming growth factor.

Table 2 Baseline and follow-up values of echocardiographic characteristics of the study group

\begin{tabular}{|c|c|c|c|c|c|c|c|}
\hline \multirow[b]{2}{*}{ Characteristic } & \multicolumn{3}{|c|}{ Spironolactone $(n=58)$} & \multicolumn{3}{|c|}{ Placebo $(n=55)$} & \multirow{2}{*}{$\begin{array}{l}\text { p Value } \\
\Delta \text { Spironolactone } \\
\text { vs } \Delta \text { Placebo }\end{array}$} \\
\hline & Baseline & Follow-up & $p$ Value & Baseline & Follow-up & $p$ Value & \\
\hline LV end-diastolic dimension, mm & $52.3 \pm 5.5$ & $52.2 \pm 5.2$ & 0.78 & $51.4 \pm 4.5$ & $51.6 \pm 3.8$ & 0.57 & 0.57 \\
\hline IVS, mm & $11.9 \pm 1.1$ & $11.3 \pm 1.1$ & 0.001 & $11.9 \pm 1.3$ & $11.7 \pm 1.4$ & 0.28 & 0.16 \\
\hline $\mathrm{PW}, \mathrm{mm}$ & $9.9 \pm 1.1$ & $9.5 \pm 1.0$ & 0.05 & $9.7 \pm 1.4$ & $9.7 \pm 1.5$ & 0.86 & 0.08 \\
\hline LV mass index, $\mathrm{g} / \mathrm{m}^{2.7}$ & $57.8 \pm 10.5$ & $54.3 \pm 10.1$ & 0.003 & $56.5 \pm 10.3$ & $56.3 \pm 11.3$ & 0.79 & 0.02 \\
\hline Left atrial dimension, $\mathrm{mm}$ & $43.5 \pm 4.7$ & $42.3 \pm 4.1$ & 0.01 & $42.8 \pm 4.4$ & $42.7 \pm 4.4$ & 0.81 & 0.04 \\
\hline$E / e^{\prime}$ & $12.3 \pm 3.0$ & $11.2 \pm 3.0$ & 0.007 & $11.4 \pm 2.6$ & $11.3 \pm 2.9$ & 0.55 & 0.05 \\
\hline E/A & $0.98 \pm 0.25$ & $0.99 \pm 0.23$ & 0.64 & $0.97 \pm 0.17$ & $0.99 \pm 0.21$ & 0.26 & 0.61 \\
\hline DT, ms & $229 \pm 44$ & $231 \pm 40$ & 0.79 & $238 \pm 41$ & $236 \pm 40$ & 0.74 & 0.66 \\
\hline $\mathrm{Sm}, \mathrm{cm} / \mathrm{s}$ & $5.8 \pm 1.2$ & $6.2 \pm 1.3$ & 0.006 & $6.0 \pm 1.2$ & $5.9 \pm 1.1$ & 0.37 & 0.001 \\
\hline $\mathrm{Em}, \mathrm{cm} / \mathrm{s}$ & $5.7 \pm 1.4$ & $6.6 \pm 1.4$ & 0.0001 & $6.0 \pm 1.4$ & $5.9 \pm 1.3$ & 0.46 & 0.0001 \\
\hline Strain, \% & $17.4 \pm 2.3$ & $19.9 \pm 2.2$ & 0.0001 & $17.6 \pm 2.1$ & $17.5 \pm 2.0$ & 0.44 & 0.0001 \\
\hline $\mathrm{SR}, 1 / \mathrm{s}$ & $1.42 \pm 0.21$ & $1.60 \pm 0.20$ & 0.0001 & $1.43 \pm 0.20$ & $1.43 \pm 0.18$ & 0.87 & 0.0001 \\
\hline IB AS, dB & $-14.3 \pm 5.8$ & $-16.9 \pm 5.1$ & 0.004 & $-15.9 \pm 5.3$ & $-16.1 \pm 5.1$ & 0.82 & 0.02 \\
\hline IB post, $\mathrm{dB}$ & $-25.0 \pm 5.5$ & $-27.6 \pm 5.5$ & 0.004 & $-24.9 \pm 6.2$ & $-25.1 \pm 5.5$ & 0.71 & 0.03 \\
\hline
\end{tabular}

\footnotetext{
Values are mean \pm SD. $\Delta$ Placebo indicates change in the placebo group during follow-up, and $\Delta$ Spironolactone the change in the spironolactone group during follow-up.
}

DT, diastolic flow wave; E/A, the ratio of peak early to late diastolic flow velocities; E/e', ratio of mitral inflow early diastolic velocity to peak early diastolic mitral annular velocity;

Em, myocardial peak early diastolic velocity; IB AS, calibrated integrated backscatter in the basal anteroseptum; IB post, calibrated integrated backscatter in the basal posterior wall; IVS, interventricular septal thickness; LV, left ventricular; PW, LV posterior wall thickness; Sm, peak systolic velocity; SR, peak systolic strain rate. 


\section{Cardiac dimensions and LV mass}

Significant reductions in LV mass index, interventricular septum and posterior wall thicknesses, and left atrial diameter at the end of the follow-up were found in the spironolactone group (table 2). All indices of cardiac function and morphology assessed by echocardiography did not alter over the observation period in the placebo group.

\section{Response to spironolactone therapy according to baseline PICP and PIIINP tertiles}

The extent of improvement with intervention in LV systolic (increase in strain) and diastolic (increase in Em and decrease in $\mathrm{E} / \mathrm{e}^{\prime}$ ) function, as well as in collagen metabolism (decrease in PICP and PIIINP), was significantly larger in patients with the highest baseline fibrosis intensity-that is, with PICP from the 3rd tertile and PIIINP from the 3rd tertile-compared with the remainder of the subjects (figure 1).

\section{Relations of changes in cardiac and fibrosis markers}

The independent predictors of improvement in LV systolic function (as indicated by an increase in strain, peak systolic strain rate and Em and a decrease in $\mathrm{E} / \mathrm{e}^{\prime}$ ) in the spironolactone group were baseline LV performance, baseline PICP, and changes at follow-up in PICP, PIIINP and myocardial reflectivity expressed by calibrated IB in the basal posterior wall. The set of variables tested in these models in different combinations also included age, baseline BMI, HOMA-IR (homoeostasis model of assessment-insulin resistance), PIIINP and calibrated IB in the basal posterior wall, changes at follow-up in blood pressure, and baseline values and follow-up changes in TGF- $\beta_{1}$ (table 3 ).

\section{DISCUSSION}

This randomised placebo-controlled study revealed that, in patients with obesity alone-that is, in the absence of any coexisting disease conditions-AA improves LV function and myocardial acoustic properties, and reduces circulating procollagen peptide levels representing collagen metabolism. Beneficial changes in cardiac performance are independently associated with the extent of baseline LV dysfunction and baseline abnormalities as well as treatment-induced improvement in fibrosis markers. While previous work has shown positive effects of AA in hypertensive heart disease and the metabolic syndrome (in the latter added as an adjunctive therapy to the drugs antagonising angiotensin II), ${ }^{16}{ }^{19}$ the important novel information provided by our investigation is that AA may favourably modify LV functional and structural characteristics in 'pure' obesity. This suggests that there is a beneficial effect independent of hypertension and diabetes-comorbidities contributing to cardiac derangements in weight excess.

\section{Pathogenesis of fibrosis in obesity}

Cardiovascular disease in obesity is multifactorial in origin. In the vast spectrum of contributors including metabolic, inflammatory-cardiodepressant, neurohormonal and haemodynamically overloading factors, increased myocardial fibrosis is thought to play a crucial role. ${ }^{8}$ 20-22 The mechanisms accounting for increased collagen turnover involve promotion of the effects of angiotensin II (via TGF- $\beta_{1}$ and aldosterone stimulation) and aldosterone (through direct agonising mineralocorticoid receptor and TGF- $\beta_{1}$ mediation). ${ }^{23-25}$

Aldosterone, being a vital component of this pathogenetic network, represents a feasible target for therapeutic interventions to limit fibrosis. Previous investigations have shown that the suppression of aldosterone-induced collagen formation may be the mainstay of clinical benefit of AA in heart failure, ${ }^{13}{ }^{14}$ and treatment with drugs opposing aldosterone provides regression of myocardial fibrosis with parallel amelioration of LV dysfunction. $^{12}$
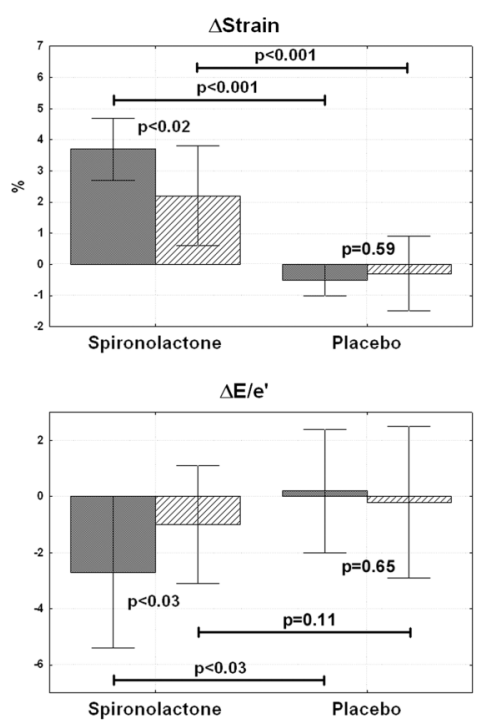
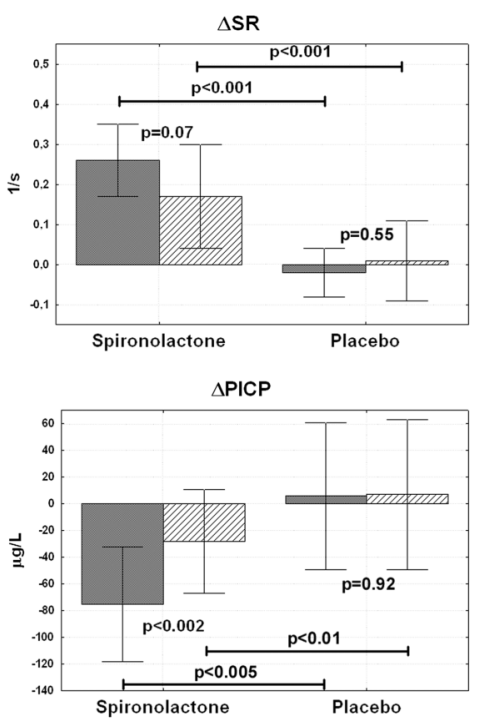

Group A

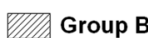
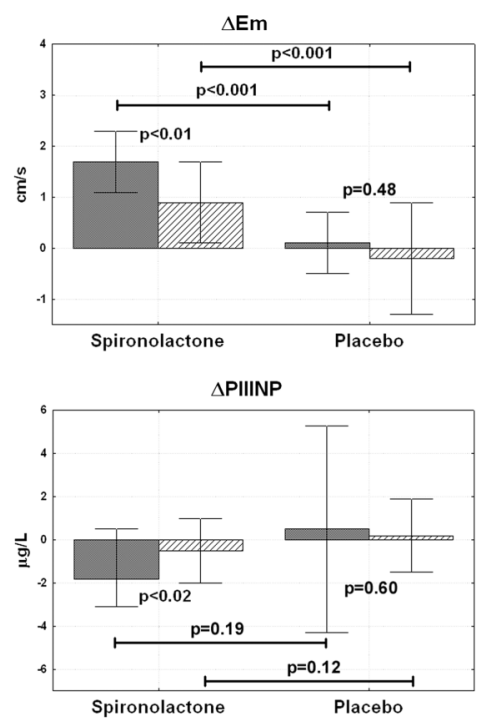

Figure 1 Response of left ventricular (LV) function and serological markers of fibrosis to spironolactone therapy in relation to baseline intensity of fibrotic processes (post hoc analysis). Group A, Patients with baseline procollagen type I C-terminal propeptide (PICP) from the 3rd tertile and baseline procollagen type III N-terminal propeptide (PIIINP) from the 3rd tertile ( $n=9$ in the spironolactone group, and $n=8$ in the placebo group). Group $B$, the remaining patients not satisfying the criteria of group $A$ ( $n=49$ in the spironolactone group, and $n=47$ in the placebo group). $\Delta$, change with intervention; E/e', ratio of mitral inflow early diastolic velocity to peak early diastolic mitral annular velocity; Em, myocardial peak early diastolic velocity; SR, peak systolic strain rate. 
Table 3 Multivariable predictors of improvement in LV function with spironolactone therapy

\begin{tabular}{|c|c|c|}
\hline & $\boldsymbol{\beta}$ & p Value \\
\hline \multicolumn{3}{|l|}{$\Delta$ Strain $\left(R^{2}=0.32\right)$} \\
\hline Baseline strain & -0.43 & 0.001 \\
\hline$\Delta \mathrm{IB}$ post & 0.26 & 0.02 \\
\hline Baseline PICP & 0.24 & 0.04 \\
\hline \multicolumn{3}{|l|}{$\Delta S R\left(R^{2}=0.40\right)$} \\
\hline Baseline SR & -0.58 & 0.001 \\
\hline$\Delta \mathrm{PIIINP}$ & -0.21 & 0.04 \\
\hline \multicolumn{3}{|l|}{$\Delta \mathrm{Em}\left(\mathrm{R}^{2}=0.35\right)$} \\
\hline Baseline Em & -0.44 & 0.001 \\
\hline Baseline PICP & 0.35 & 0.002 \\
\hline BMI & 0.20 & 0.07 \\
\hline \multicolumn{3}{|l|}{$\Delta \mathrm{E} / \mathrm{e}^{\prime}\left(\mathrm{R}^{2}=0.19\right)$} \\
\hline Baseline E/e' & -0.35 & 0.005 \\
\hline$\triangle \mathrm{PICP}$ & 0.25 & 0.04 \\
\hline
\end{tabular}

\section{Treatment responses of fibrosis in obesity}

The present study extended the observations concerning favourable cardiovascular effects of AA on patients with obesity and abnormal LV performance. In line with prior studies in other disease populations, ${ }^{12} 16$ we found both improvement in LV function and a decrease in serological fibrosis markers-PICP and PIIINP-with intervention. Procollagen peptides are released during collagen biosynthesis and, as evidenced, after exclusion of non-cardiac disorders promoting fibrosis, their blood pool reflects collagen turnover in the myocardial compartment, also including treatment-induced changes. ${ }^{26-29}$ In contrast with PICP and PIIINP, the decrease in fibrosis mediator-TGF- $\beta_{1}$ with AA (congruent with analogous explorations in animals)-was not an independent determinant of favourable changes in LV disturbances. The improvement in myocardial reflectivity, assessed by calibrated IB-an echo parameter validated to correspond to myocardial collagen content-supports the beneficial impact of spironolactone on fibrous tissue deposition. Evidence of cardiac fibrosis regression ( $\Delta$ calibrated IB) is independently associated with LV functional improvement (increase in strain) at follow-up.

Notwithstanding the analysis revealing large or medium effect size of post-therapy improvements, the absolute changes in cardiac function and fibrotic markers were rather limited. This might be associated with the less extensive progression of LV/ fibrosis abnormalities in the population of obese subjects without comorbidities enhancing cardiovascular and fibrosisrelated pathologies.

The association of baseline LV performance and PICP with treatment response indicates that greater benefits with AA may be expected in obese patients with more severe pretreatment LV dysfunction and fibrosis. The significance of baseline fibrosis intensity in predicting the outcome of AA treatment was corroborated by post hoc analysis based on tertiles of PICP and PIIINP, and this is consistent with previous reports showing the predominance of salutary effects of spironolactone therapy in patients with higher circulating procollagen peptides and increased collagen accumulation in the myocardium. ${ }^{12} 13$

Fat distribution was not an independent correlate of the demonstrated cardiac improvements. However, the absence of association with central adiposity (which is a source of profibrotic compounds), nor the android-to-gynoid fat ratio (which describes the balance between deleterious and postulated cardioprotective deposits), does not negate the potential roles of both fat depots in eliciting the response to antifibrotic treatment, but might be attributable to a narrow range of these variables in our selected population. Moreover, the mechanism of the effect of spironolactone on LV morphology is unclear, particularly in relation to the reduction in LV hypertrophy.

\section{Limitations}

This study has a variety of limitations. First, we used echocardiographic indices of LV function to substitute for clinical end points. However, the relationships of these surrogates to heart failure symptoms have been evidenced in a multitude of prior investigations. Second, the extent of myocardial fibrosis was not directly estimated by the morphometry, but this would not be ethically justified in our patients. The use of gadolinium late enhancement might have improved the accuracy of estimation of cardiac fibrosis, but it should be remembered that the fibrotic process in obesity is diffuse, and a process such as T1 mapping would probably be required, which was unavailable. Third, despite their association with myocardial fibrosis, circulating procollagen peptides are not specific to the heart and may be influenced by other extracardiac sources. In contrast with PICP, which is released exclusively during the conversion of procollagen type I into collagen type I, PIIINP may be derived from both collagen synthesis and degradation and cannot be regarded as a 'pure' indicator of anabolic processes in fibrous tissue. ${ }^{30}$ Finally, we cannot definitely rule out the contribution of the hypotensive effect of AA to the improvements in cardiac structure and function. However, the independence of these responses from changes in blood pressure suggests that these haemodynamic effects are not of primary importance.

\section{Conclusions and implications}

AA in obese patients without other comorbidities ameliorates LV functional and structural impairments, and some of these effects may be associated with the concomitant limitation of excessive turnover of extracellular matrix. Our data suggest that treatment with mineralocorticoid receptor antagonists could be considered in obese patients with decreased cardiac performance and enhanced collagen synthesis, implying that a selection process would be needed to justify the implementation of this pharmacotherapy. The present study sets the stage for larger-scale clinical trials designed to verify our findings against clinical end points, as well as to evaluate the outcomes of aldosterone blockade in obesity over a longer duration.

Contributors WK: study conception and design, acquisition of data, statistical analysis, analysis and interpretation of data, drafting of the manuscript, and final approval of the manuscript. WK takes primary responsibility for the data. MP-K: acquisition of data, statistical analysis, analysis and interpretation of data, drafting of the manuscript, and final approval of the manuscript. HS-O: acquisition of data, statistical analysis, analysis and interpretation of data, drafting of the manuscript, and final approval of the manuscript. AM: analysis and interpretation of data, revising critically the manuscript for important intellectual content, and final approval of the manuscript. THM: study conception and design, analysis and interpretation of data, revising critically the manuscript for important intellectual content, and final approval of the manuscript.

Funding This work was supported in part by a grant from the National Health and Medical Research Council of Australia (455832).

Competing interests None.

Patient consent Obtained. 
Ethics approval HREC of Princess Alexandra Hospital Brisbane and Wroclaw Medical University, Poland.

Provenance and peer review Not commissioned; externally peer reviewed.

\section{REFERENCES}

1 Gregg EW, Cheng YJ, Cadwell BL, et al. Secular trends in cardiovascular disease risk factors according to body mass index in US adults. JAMA 2005;293:1868-74.

2 Prevalence of overweight, obesity, and extreme obesity among adults: United States, trends 1960-1962 through 2007-2008. June 2010. http://www.cdc.gov/ nchs/data/hestat/obesity_adult_07_08/obesity_adult_07_08.htm (accessed 29 May 2012).

3 Kenchaiah S, Evans JC, Levy $\mathrm{D}$, et al. Obesity and the risk of heart failure. $\mathrm{N}$ Engl $\mathrm{J}$ Med 2002:347:305-13.

4 Flegal KM, Graubard BI, Williamson DF, et al. Cause-specific excess deaths associated with underweight, overweight, and obesity. JAMA 2007:298:2028-37.

5 Pischon $\mathrm{T}$, Boeing $\mathrm{H}$, Hoffmann $\mathrm{K}$, et al. General and abdominal adiposity and risk of death in Europe. N Engl J Med 2008;359:2105-20.

6 Di Bello V, Santini F, Di Cori A, et al. Relationship between preclinical abnormalities of global and regional left ventricular function and insulin resistance in severe obesity: a color Doppler imaging study. Int J Obes (Lond) 2006:30:948-56.

7 Wong CY, O'Moore-Sullivan T, Leano R, et al. Alterations of left ventricular myocardial characteristics associated with obesity. Circulation 2004:110:3081-7.

8 Kosmala W, Plaksej R, Przewlocka-Kosmala M, et al. Matrix metalloproteinases 2 and 9 and their tissue inhibitors 1 and 2 in premenopausal obese women: relationship to cardiac function. Int J Obes (Lond) 2008;32:763-71.

9 Quilliot D, Alla F, Böhme $P$, et al. Myocardial collagen turnover in normotensive obese patients: relation to insulin resistance. Int J Obes (Lond) 2005:29:1321-8.

10 Leopoldo AS, Sugizaki MM, Lima-Leopoldo AP, et al. Cardiac remodeling in a rat model of diet-induced obesity. Can J Cardiol 2010:26:423-9.

11 Zaman AK, Fujii S, Goto D, et al. Salutary effects of attenuation of angiotensin II on coronary perivascular fibrosis associated with insulin resistance and obesity. J Mol Cell Cardiol 2004;37:525-35.

12 Izawa $\mathrm{H}$, Murohara T, Nagata $\mathrm{K}$, et al. Mineralocorticoid receptor antagonism ameliorates left ventricular diastolic dysfunction and myocardial fibrosis in mildly symptomatic patients with idiopathic dilated cardiomyopathy: a pilot study. Circulation 2005;112:2940-5.

13 Zannad F, Alla F, Dousset B, et al. Limitation of excessive extracellular matrix turnover may contribute to survival benefit of spironolactone therapy in patients with congestive heart failure: insights from the randomized aldactone evaluation study (RALES). Rales Investigators. Circulation 2000;102:2700-6.

14 Iraqi W, Rossignol P, Angioi M, et al. Extracellular cardiac matrix biomarkers in patients with acute myocardial infarction complicated by left ventricular dysfunction and heart failure: insights from the Eplerenone Post-Acute Myocardial Infarction Heart Failure Efficacy and Survival Study (EPHESUS) study. Circulation 2009;119:2471-9.
15 Zannad F, McMurray JJ, Krum H, et al. the EMPHASIS-HF Study Group. Eplerenone in patients with systolic heart failure and mild symptoms. N Engl J Med 2011:364:11-21.

16 Kosmala W, Przewlocka-Kosmala M, Szczepanik-Osadnik H, et al. Beneficial effect of aldosterone antagonism on left ventricular function and structure and fibrosis markers in the metabolic syndrome-a randomized trial. J Am Coll Cardiol Cardiovasc Imaging 2011;4:1239-49.

17 Schulz KF, Altman DG, Moher D; CONSORT Group. CONSORT 2010 statement: updated guidelines for reporting parallel group randomized trials. PLoS Med 2010;7:e1000251.

18 Lang RM, Bierig M, Devereux RB, et al. Chamber Quantification Writing Group; American Society of Echocardiography's Guidelines and Standards Committee; European Association of Echocardiography. Recommendations for chamber quantification: a report from the American Society of Echocardiography's Guidelines and Standards Committee and the Chamber Quantification Writing Group, developed in conjunction with the European Association of Echocardiography. J Am Soc Echocardiogr 2005;18:1440-63.

19 Mottram PM, Haluska B, Leano $\mathrm{R}$, et al. Effect of aldosterone antagonism on myocardial dysfunction in hypertensive patients with diastolic heart failure. Circulation 2004;110:558-65

20 Di Bello V, Santini F, Di Cori A, et al. Obesity cardiomyopathy: is it a reality? An ultrasonic tissue characterization study. J Am Soc Echocardiogr 2006:19:1063-71.

21 Vasan RS. Cardiac function and obesity. Heart 2003;89:1127-9.

22 Peterson LR, Herrero $\mathrm{P}$, Schechtman $\mathrm{KB}$, et al. Effect of obesity and insulin resistance on myocardial substrate metabolism and efficiency in young women. Circulation 2004;109:2191-6.

23 Laviades C, Varo N, Díez J. Transforming growth factor beta in hypertensives with cardiorenal damage. Hypertension 2000;36:517-22.

24 Robert V, Silvestre JS, Charlemagne D, et al. Biological determinants of aldosterone-induced cardiac fibrosis in rats. Hypertension 1995;26:971-8.

25 Brilla CG. Renin-angiotensin-aldosterone system and myocardial fibrosis. Cardiovasc Res 2000;47:1-3.

26 Díez J, Laviades C. Monitoring fibrillar collagen turnover in hypertensive heart disease. Cardiovasc Res 1997;35:202-5.

27 López B, Querejeta R, Varo N, et al. Usefulness of serum carboxy-terminal propeptide of procollagen type I in assessment of the cardioreparative ability of antihypertensive treatment in hypertensive patients. Circulation 2001;104:286-91.

28 Díez J, Panizo A, Gil MJ, et al. Serum markers of collagen type I metabolism in spontaneously hypertensive rats: relation to myocardial fibrosis. Circulation 1996:93:1026-32.

29 Uusimaa P, Risteli J, Niemelä $\mathrm{M}$, et al. Collagen scar formation after acute myocardial infarction: relationships to infarct size, left ventricular function, and coronary artery patency. Circulation 1997;96:2565-72.

30 Peuhkurinen KJ, Risteli L, Melkko JT, et al. Thrombolytic therapy with streptokinase stimulates collagen breakdown. Circulation 1991;83:1969-75. 